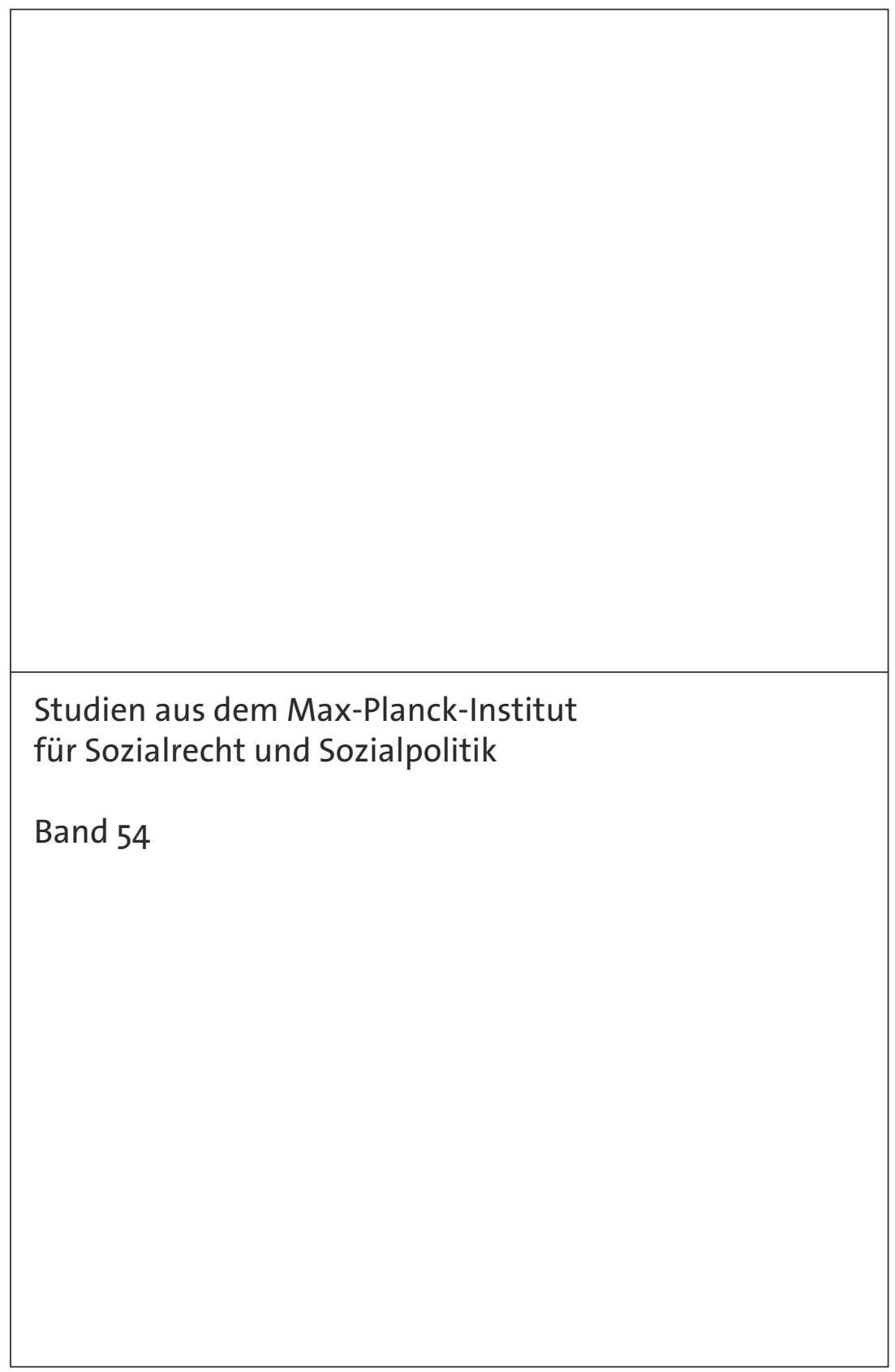


Prof. Dr. Ulrich Becker, LL.M./

Nikola Wilman, LL.M., MJur. (Hrsg.)

\section{Im Zweifel auf Privatrezept?}

Sozial- und haftungsrechtliche Aspekte des Off-Label-Use 
Die Deutsche Nationalbibliothek verzeichnet diese Publikation in der Deutschen Nationalbibliografie; detaillierte bibliografische Daten sind im Internet über http://dnb.d-nb.de abrufbar.

ISBN 978-3-8329-7282-0

\section{Auflage 2012}

(c) Nomos Verlagsgesellschaft, Baden-Baden 2012. Printed in Germany. Alle Rechte, auch die des Nachdrucks von Auszügen, der fotomechanischen Wiedergabe und der Übersetzung, vorbehalten. Gedruckt auf alterungsbeständigem Papier. 


\section{Vorwort}

Im Jahr 2010 hat das Max-Planck-Institut für ausländisches und internationales Sozialrecht, nun nach der Umbenennung im Jahre 2011 das Max-Planck-Institut für Sozialrecht und Sozialpolitik, eine Tagung zu den sozial- und haftungsrechtlichen Folgen des Off-Label-Use veranstaltet. Da sowohl die Tagung als auch die dort gehaltenen Referate auf ein breites Interesse gestoßen sind, haben sich Referenten und Herausgeber dazu entschlossen, die Beiträge in aktualisierter Form und leicht veränderter Zusammensetzung in diesem Tagungsband abzudrucken.

Der „Off-Label-Use“ und damit das Thema, dem die Beiträge gewidmet sind, ist für Sozialrechtler relativ neu. Noch Anfang des Jahrtausends hätten sich die meisten Leser gefragt, was darunter überhaupt zu verstehen ist. Der Begriff, der sich auf dem USamerikanischen Pharmamarkt entwickelt hat, war nur wenigen Eingeweihten bekannt. Mittlerweile ist er gängig und hat eine steile Karriere hinter sich gebracht. Er bezeichnet im Grunde genommen eine eigene und fast schon etablierte Kategorie der Versorgung mit Arzneimitteln. Dennoch sind die Fragen um die Voraussetzungen der Versorgung und selbst um die Zulässigkeit dieser Kategorie nicht endgültig geklärt. Und sie sind heute aktuell wie nie zuvor, was sowohl an den Entwicklungen in der Rechtsprechung als auch im Recht der gesetzlichen Krankenversicherung liegt. Dabei sind alle durch das sog. Leistungsdreieck verbundenen Personen betroffen: die Patienten, um deren Rechte auf eine notwendige Arzneimittelversorgung es geht, die Ärzte, die sich beim OffLabel-Use zwischen Arzneimittelregreß und zivilrechtlicher Haftung bewegen, und die Pharmaunternehmen, die auch Strategien für die Zulassung ihrer Arzneimittel verfolgen können.

Ausgangspunkt ist immer der sozialrechtliche Rahmen für die Erbringung von Arzneimitteln. Das Sozialrecht knüpft an die Arzneimittelzulassung an, es nimmt dessen Voraussetzungen hin und ebenso die Funktion des Arzneimittelrechts, einen Marktzugang zu regeln. Andererseits besteht die medizinische Notwendigkeit, Arzneimittel auBerhalb ihrer indikationsbezogenen Zulassung anzuwenden: Sei es, weil zugelassene Arzneimittel nicht vertragen werden, sie im Einzelfall nicht wirksam oder gar keine Arzneimittel verfügbar sind. Gerade in der Kinderheilkunde und in der Onkologie, aber auch bei der Behandlung spezieller Krankheiten wie bestimmten Formen der Multiplen Sklerose, ist der Off-Label-Use fester Bestandteil des „medizinischen Alltags“.

Geebnet hat den Weg die Rechtsprechung, und zwar das BSG mit mehreren grundlegenden Entscheidungen vor allem einer Entscheidung vom 19.3.2002 (Sandoglobulin). Danach waren drei Voraussetzungen für einen Off-Label-Use zu prüfen: (1) Die Krankheit muss schwerwiegend sein; (2) es darf keine andere Behandlungsmöglichkeit bestehen, (3) und es muss begründete Aussicht auf einen Behandlungserfolg geben, zugleich verstanden als Zulassungsfähigkeit des Arzneimittels. Es folgte die Entscheidung des BVerfG vom 6.12.2005, mit der das Konzept zugunsten der Behandlung von lebensbe- 
drohlicher Krankheit im Einzelfall erweitert worden ist. Der Gesetzgeber hat dann reagiert durch die Einfügung des $\S 35 \mathrm{~b}$ Abs. 3 SGB V (jetzt: $\S 35 \mathrm{c}$ Abs.1 SGB V) mit der Möglichkeit, Expertengruppen beim BfArM einzusetzen, deren Empfehlungen über den GBA Eingang in die Arzneimittelrichtlinie finden und eine Art von Positivliste begründen könnten.

Offen bleibt dennoch eine Reihe von Fragen: Wäre es nicht sinnvoll, statt auf Alternativlosigkeit eines Arzneimittels als Versorgungsvoraussetzung auf die Wirtschaftlichkeit abzustellen? Welche Anforderungen sollen für den Nachweis von möglichen Behandlungserfolgen gelten? Das BSG hat seine Rechtsprechung zudem zwischenzeitlich weiterentwickelt. Welche Spielräume bleiben für eine richterliche Rechtsfortbildung angesichts der gesetzgeberischen Reformen? Wird diese durch die Aufnahme von Empfehlungen zur Off-Label- Anwendung von Arzneimitteln begrenzt? Wie viel Raum bleibt, wenn es (a) Expertengruppen überhaupt nur gibt, und (b), wenn diese Entscheidungen getroffen haben - sind eventuell die Grundsätze, die für die Zulassung vom Therapien durch den GBA gelten entsprechend anwendbar? Welche Rolle spielt eine verfassungskonforme Auslegung?

Das sind nur einige Fragen, die in den folgenden Beiträgen behandelt werden. Der Band beginnt im Sinne einer tatsächlichen Grundlegung mit einer Darstellung des OffLabel Use aus ärztlicher Sicht im Bereich der Onkologie (Dirk Jäger), die neben der Pädiatrie eines der Hauptanwendungsgebiete des Off-Label-Use darstellt. Der zweite Beitrag geht auf zwei ausgewählte Aspekte des Off-Label-Use in den USA ein (Nikola Wilman), dem Land, in dem der Begriff des „Off-Label-Use“ seinen Ursprung hat. Es folgt eine eingehende Aufarbeitung der Rechtsprechung des Bundessozialgerichts zum Off-Label-Use und deren Entwicklung, ausgehend vom Remedacen-Urteil über die SKAT-Entscheidung bis hin zum Sandoglobulin-Urteil vom 19.3.2002 (Hans-Jürgen Kretschmer). Aus Sicht der Rechtsandwendung werden im Anschluss die durch das BSG aufgestellten Kriterien beleuchtet (Julia Wicke) und deren Praktikabilität im alltäglichen Umgang mit Patienten, die sich häufig einem sehr hohen Leidensdruck ausgesetzt sehen, hinterfragt. Es folgt ein Statement zum Off-Label-Use aus Sicht der Pharmaindustrie, das insbesondere kritisch auf das Verfahren zur Aufnahme von Off-LabelIndikationen in die entsprechende Anlage der Arzneimittel-Richtlinie des GBA eingeht (Barbara Sickmüller und Christine Lietz) Der Band schließt mit einem Beitrag zu den zivil-, sozial- und strafrechtlichen Haftungsrisiken, denen sich ein Arzt, der den OffLabel-Use eines Arzneimittels verordnet, ausgesetzt sieht (Ute Walter).

Mein Dank gilt den Autoren der Beiträge, Nikola Wilman für die Organisation der Tagung und die Vorbereitung der Publikation, Stefan Stegner dafür, dass er die Texte in die richtige Form gebracht hat, und allen Mitarbeiterinnen und Mitarbeitern des Instituts, die bei der Durchführung der Tagung geholfen haben. 


\section{Inhaltsverzeichnis}

Teil 1: Die (Rechts)-problematik des Off-Label-Use

Off-Label-Use in der Onkologie

Dirk Jäger

Off-Label-Use in den USA

Nikola Wilman

Teil 2: Sozialrechtliche Aspekte des Off-Label-Use

Aktuelle Rechtsprechung zum Off-Label-Use

Hans-Jürgen Kretschmer

Der Off-Label-Use - Probleme bei der Rechtsanwendung

Julia Wicke

Der Off-Label-Use aus Sicht der Pharmaindustrie

Barbara Sickmüller und Christine Lietz

Teil 3: Haftungsrechtliche Aspekte des Off-Label-Use

Ärzte und ihre Verordnung von Arzneimitteln im Off-Label-Use Ute Walter

Autorenverzeichnis 
